\title{
A Priori Bounds for Periodic Solutions to Nonlinear Differential Equation with Delay
}

\author{
Xinyi Chen
}

China Minorities Information Technology Institute, Northwest University for Nationalities, Lanzhou 730030 P. R. China

\begin{abstract}
A priori bounds are established for periodic solutions of a nonlinear differential equation with delay. By means of these bounds, an existence theorem for periodic solutions can be obtained by means of Mawhin's continuation theorem. Our work generalizes the known result.
\end{abstract}

keywords-priori bound; nonlinear differential equation; periodic solution; coincidence degree

\section{INTRODUCTION}

In [1], continuation theorems were introduced and applied to the existence of solutions of nonlinear equation. In particular, a specific example was given in [1](see also [2]) on how periodic solutions can be obtained by means of these theorems for the nonlinear equation. $x^{\prime \prime}(t)+f\left(x^{\prime}(t)\right)+h(t, x(t))=0$, where $f$ is a real continuous function defined on $R$ such that $f(0)=0, h$ is a real continuous function defined on $R \times R, 2 \pi$-periodic in $t$ and $h(t, x) x<0$ for $|x| \geq r$ and $t \in[0,2 \pi]$. In the course of derivations, it is realized that once appropriate a priori bounds for the $2 \pi$-periodic solutions of the following equation $x^{\prime \prime}(t)+\lambda f\left(x^{\prime}(t)\right)+\lambda h(t, x(t))=0$ are known for each $\lambda \in(0,1)$, then standard procedures will allow these theorems to imply existence of periodic solutions to the nonlinear equation.

In this paper, we will be concerned with a similar equation with an additional delay

$$
x^{\prime \prime}(t)+\lambda f\left(x^{\prime}(t)\right) x(t)+\lambda g(x(t-\tau(t)))=\lambda p(t), \lambda \in(0,1)
$$

where $f, g, p$ and $\tau$ are real continuous functions defined on $R$ such that $f(0)=0, \tau$ and $P$ are periodic with period $2 \pi$, and $\int_{0}^{2 \pi} p(t) d t=0$.We will establish a priori bounds for solutions of this equation under relatively simple conditions on $f$ and $g$. Once these bounds are obtained, existence of periodic solutions can be demonstrated for the equation

$$
x^{\prime \prime}(t)+f\left(x^{\prime}(t)\right) x(t)+g(x(t-\tau(t)))=p(t)
$$

We remark that there are a number of studies which are concerned with the existence of periodic solutions of differential equations by means of establishing a priori bounds (see [3]). Other related results can be found in [4-7].

\section{MAIN RESULTS}

Theorem 1Suppose there are positive constants $M, D$, $a<1$, and nonnegative constant $K$ such that

$$
\begin{aligned}
& \text { [A] }|f(x)| \leq a K \text {, for any } x \in R, \\
& \text { [B] } x g(x)>0 \text { and }|g(x)|>K|x| \text { for }|x| \geq D,
\end{aligned}
$$$$
\text { [C] } \lim _{x \rightarrow-\infty} \frac{g(x)}{x} \leq M \text {, and }
$$

[D] $8 \pi^{2}\left(\frac{a K}{1-a}+\frac{M}{1-a}\right)<1$. Then for any $2 \pi$-periodic solution $\quad x=x(t) \quad$ of $\quad(1), \quad\left|x^{\prime}(t)\right| \leq D_{2} \quad$ and $|x(t)| \leq \frac{D}{1-a}+\frac{2 \pi D_{2}}{1-a}$ for some positive number $D_{2}$.Proof Let $x=x(t)$ be a $2 \pi$-periodic solution of the equation (1). Since $x(0)=x(2 \pi)$, there is some $t_{0} \in[0,2 \pi]$ such that $x^{\prime}\left(t_{0}\right)=0$. In view of (1), we see that for any $t \in[0,2 \pi]$,

$$
\begin{gathered}
\left|x^{\prime}(t)\right|=\left|\int_{t_{0}}^{t} x^{\prime \prime}(s) d s\right| \leq \int_{0}^{2 \pi}\left|x^{\prime \prime}(s)\right| d s \\
\leq \lambda \int_{0}^{2 \pi}\left|f\left(x^{\prime}(s)\right) x(s)\right| d s+\lambda \int_{0}^{2 \pi} \mid g(x(s \\
-\tau(s)))\left|d s+\lambda \int_{0}^{2 \pi}\right| p(s) \mid d s \\
\leq 2 a \pi K \max _{0 \leq s \leq 2 \pi}|x(s)|+\int_{0}^{2 \pi}|g(x(s-\tau(s)))| d s \\
+2 \pi \max _{0 \leq s \leq 2 \pi}|p(s)|
\end{gathered}
$$


We assert that

$$
\begin{aligned}
& \int_{0}^{2 \pi}|g(x(t-\tau(t)))| d t \leq 2 a \pi K \max _{0 \leq t \leq 2 \pi}|x(t)| \\
+ & 4 \pi(M+\varepsilon) \max _{0 \leq t \leq 2 \pi}|x(t)|+4 \pi g_{\rho}
\end{aligned}
$$

for some positive numbers $\rho, \varepsilon$ and $g_{\rho}=\max _{|t| \leq \rho}|g(t)|$. Indeed, integrating equation (1) from 0 to $2 \pi$, we see that

$$
\int_{0}^{2 \pi}\left\{f\left(x^{\prime}(t)\right) x(t)+g(x(t-\tau(t)))\right\} d t=0
$$

Thus there is some $t_{1} \in[0,2 \pi]$ such that

$$
f\left(x^{\prime}\left(t_{1}\right)\right) x\left(t_{1}\right)=-g\left(x\left(t_{1}-\tau\left(t_{1}\right)\right)\right) .
$$

$10 K=0$. If $\left|x\left(t_{1}-\tau\left(t_{1}\right)\right)\right|>D$, [A] and [B] implies that. This contradiction establishes $\left|x\left(t_{1}-\tau\left(t_{1}\right)\right)\right| \leq D .20$ $K>0$. If $\left|x\left(t_{1}-\tau\left(t_{1}\right)\right)\right|>D$, noting condition [A] and [B], we see that

$$
\begin{aligned}
& K\left|x\left(t_{1}-\tau\left(t_{1}\right)\right)\right| \varangle g\left(x\left(t_{1}-\tau\left(t_{1}\right)\right)\right) \mid \\
= & \left|f\left(x^{\prime}\left(t_{1}\right)\right) x\left(t_{1}\right)\right| \leq a K\left|x\left(t_{1}\right)\right| .
\end{aligned}
$$

Hence

$$
\left|x\left(t_{1}-\tau\left(t_{1}\right)\right)\right|<a\left|x\left(t_{1}\right)\right| \leq a \max _{0 \leq t \leq 2 \pi}|x(t)|
$$

Thus $K=0$ or $K>0$, we have

$$
\left|x\left(t_{1}-\tau\left(t_{1}\right)\right)\right| \leq D+a \max _{0 \leq t \leq 2 \pi}|x(t)|
$$

Since $x(t)$ is $2 \pi$-periodic, we may infer that $\left|x\left(t_{2}\right)\right| \leq D+a \max _{0 \leq t \leq 2 \pi}|x(t)|$ for some $t_{2} \in[0,2 \pi]$. We see that

$$
\begin{gathered}
|x(t)|=\left|x\left(t_{2}\right)+\int_{t_{2}}^{t} x^{\prime}(s) d s\right| \\
\leq D+a \max _{0 \leq s \leq 2 \pi}|x(s)|+\int_{0}^{2 \pi}\left|x^{\prime}(s)\right| d s, t \in[0,2 \pi],
\end{gathered}
$$

hence

$$
\max _{0 \leq t \leq 2 \pi}|x(t)| \leq \frac{D}{1-a}+\frac{1}{1-a} \int_{0}^{2 \pi}\left|x^{\prime}(s)\right| d s
$$

$$
\text { Let } F(z)=8 \pi^{2}\left(\frac{a K}{1-a}+\frac{1}{1-a}(M+z)\right), z \in[0,+\infty) \text {. From }
$$

[D] we get $F(0)<1$. Since $F$ is Continuous on $[0,+\infty)$, there is some $\delta>0$ such that

$$
F(z)=8 \pi^{2}\left(\frac{a K}{1-a}+\frac{1}{1-a}(M+z)\right)<1, z \in(0, \delta]
$$

Let $\varepsilon=\frac{\delta}{2}$, we have

$$
8 \pi^{2}\left(\frac{a K}{1-a}+\frac{1}{1-a}(M+\varepsilon)\right)<1
$$

In view of [C], there is $\rho>D$, such that

$$
\frac{g(x)}{x}<(M+\varepsilon), x<-\rho
$$

$$
\text { Let } E_{1}=\{t \in[0,2 \pi] \mid x(t-\tau(t))>\rho\} \text {, }
$$$$
E_{2}=\{t \in[0,2 \pi] \mid x(t-\tau(t))<-\rho\},
$$

$E_{3}=\{t \in[0,2 \pi] \| x(t-\tau(t)) \mid \leq \rho\}$,then from (5), we get $\left(\int_{E_{1}}+\int_{E_{2}}+\int_{E_{3}}\right) g(x(t-\tau(t))) d t \leq \int_{0}^{2 \pi}\left|f\left(x^{\prime}(t)\right) x(t)\right| d t$,and in view of

condition

[B],

$$
\int_{E_{1}}|g(x(t-\tau(t)))| d t=\int_{E_{1}} g(x(t-\tau(t))) d t
$$

$\leq\left(\int_{E_{2}}+\int_{E_{3}}\right)|g(x(t-\tau(t)))| d t+\int_{0}^{2 \pi}\left|f\left(x^{\prime}(t)\right) x(t)\right| d t$

Therefore,

$$
\begin{gathered}
\int_{0}^{2 \pi}|g(x(t-\tau(t)))| d t \leq 2 a \pi K \max _{0 \leq t \leq 2 \pi}|x(t)| \\
+4 \pi(M+\varepsilon) \max _{0 \leq t \leq 2 \pi}|x(t)|+4 \pi g_{\rho},
\end{gathered}
$$

as required. Combining (3), (4), (6), and noting condition [D], we see that

$$
\left|x^{\prime}(t)\right| \leq D_{2}, t \in[0,2 \pi]
$$

for some positive number $D_{2}$. In view of (6), we see that

$$
|x(t)| \leq \frac{D}{1-a}+\frac{1}{1-a} \int_{0}^{2 \pi}\left|x^{\prime}(t)\right| d t \leq \frac{D}{1-a}+\frac{2 \pi D_{2}}{1-a}, t \in[0,2 \pi] .
$$

\section{The proof is complete.}


Corollary 1Suppose there are positive constants $M, D$, $a<1$, and nonnegative constant $K$ such that

$$
\begin{aligned}
& \text { [A] }|f(x)| \leq a K \text {, for any } x \in R, \\
& {[\mathrm{~B}] x g(x)>0 \text { and }|g(x)|>K|x| \text { for }|x| \geq D,} \\
& \lim _{x \rightarrow+\infty} \frac{g(x)}{x} \leq M, \text {, and } \\
& \text { [D] } 8 \pi^{2}\left(\frac{a K}{1-a}+\frac{M}{1-a}\right)<1
\end{aligned}
$$

Then for any $2 \pi$-periodic solution $x=x(t)$ of (1), $\left|x^{\prime}(t)\right| \leq D_{2}$ and $|x(t)| \leq \frac{D}{1-a}+\frac{2 \pi D_{2}}{1-a}$ for some positive number $D_{2}$.

By means of the a priori bounds just obtained, we may follow the standard procedures as explained in various places of [1] and the continuation theorem in [1] to show the existence of a periodic solution of (2). For the sake of completeness, a brief sketch is included as follows.

Let $X$ be the Banach space of all continuous differentiable functions of the form $x=x(t)$, defined on $R$ such that $x(t+2 \pi)=x(t)$ for all $t$, and endowed with the norm $\|x\|_{1}=\max _{0 \leq t \leq 2 \pi}\left\{|x(t)|,\left|x^{\prime}(t)\right|\right\}$. Also let $Y$ be the Banach space of all continuous functions of the form $y=y(t)$, defined on $R$ such that $y(t+2 \pi)=y(t)$ for all $t$, and endowed with the norm $\|y\|_{0}=\max _{0 \leq t \leq 2 \pi}|y(t)|$. Finally let $\Omega$ be the subspace of $X$ containing functions of the form $x=x(t)$, such that $|x(t)|<\bar{D}$ and $\left|x^{\prime}(t)\right|<\bar{D}$, where $\bar{D}$ is a fixed number greater than $\frac{D}{1-a}+\frac{2 \pi D_{2}}{1-a}$. Now let $L$ : $X$ I $C^{2}(R, R) \rightarrow Y$ be the differential operator defined by $(L x)(t)=x^{\prime \prime}(t)$ for $t \in R$, and let $N: \quad X \rightarrow Y$ be defined by $(N x)(t)=-f\left(x^{\prime}(t)\right) x(t)-g(x(t-\tau(t)))+p(t)$, $t \in R$. Let $\operatorname{Im} L$ and $\operatorname{Ker} L$ be, respectively, the image and kernel of the operator $L$. Clearly, $\operatorname{Ker} L=R$. Furthermore, if we define the projections $P: X \rightarrow \operatorname{Ker} L$ and $Q$ : $Y \rightarrow Y / \operatorname{Im} L$ by

$$
(P x)(t)=\frac{1}{2 \pi} \int_{0}^{2 \pi} x(t) d t, \quad(Q y)(t)=\frac{1}{2 \pi} \int_{0}^{2 \pi} y(t) d t,
$$
$t \in R$, respectively, then $\operatorname{Ker} L=\operatorname{Im} P$, and $\operatorname{Ker} L=\operatorname{Im} L$. Furthermore, the operator $L$ is a Fredholm operator with index zero, and the operator $N$ is $L$ - compact on the closure $\bar{\Omega}$ of $\Omega[1]$.

For any $\lambda \in(0,1)$ and any $x=x(t)$ in the domain of $L$ which also belongs to $\partial \Omega$, we must have $L x \neq \lambda N x$. For otherwise in view of (10) and (11), we see that $X$ belongs to the interior of $\Omega$, which is contrary to the assumption that $x \in \partial \Omega$. Next, note that a function $x=x(t)$ in the intersection of $\operatorname{Ker} L$ and $\partial \Omega$ must be the constant functions $x(t)=\bar{D}$ or $x(t)=-\bar{D}$. Hence

$$
\begin{gathered}
(Q N)(x)=\frac{1}{2 \pi} \int_{0}^{2 \pi}\left(-f\left(x^{\prime}(t)\right) x(t)\right. \\
-g(x(t-\tau(t)))+p(t)) d t=-g( \pm \bar{D}) \neq 0 .
\end{gathered}
$$

Finally, consider the following mapping

$$
F(x, s)=s x+(1-s) g(x), 0 \leq s \leq 1 .
$$

Since for every $s \in[0,1]$ and $x$ in the intersection of $\operatorname{Ker} L$ and $\partial \Omega$, we have

$$
\begin{aligned}
& x F(x, s)=s x^{2}+(1-s) x g(x)>0 \\
& \text { thus } F(x, s) \text { is a homotopy. This shows that } \\
& \quad \operatorname{deg}\{Q N x, \Omega \text { I } \operatorname{Ker} L, 0\} \\
& =\operatorname{deg}\{-g(x), \Omega \text { I } \operatorname{Ker} L, 0\} \\
& =\operatorname{deg}\{-x, \Omega \text { I } \operatorname{Ker} L, 0\} \\
& =\operatorname{deg}\{-x, \Omega \text { I } R, 0\} \neq 0 .
\end{aligned}
$$

We have thus verified all the assumptions of the continuation theorem [1]. Under the assumptions in Theorem 1, equation (2) thus has a $2 \pi$-periodic solution.

As an example, consider the equation

$$
\begin{aligned}
& x^{\prime \prime}(t)+\frac{\left.\exp \left\{-x^{\prime}(t)\right)^{2}\right\} x(t)}{66 \pi^{2}}+\frac{h(x(t-\sin t))}{32 \pi^{2}}=\cos t \\
& \text { where } \\
& \qquad h(x)= \begin{cases}x^{3}, & x \geq 0, \\
x, & x<0 .\end{cases}
\end{aligned}
$$

Take

$$
f(x)=\frac{\exp \left(-x^{2}\right)}{66 \pi^{2}}, g(x)=\frac{h(x)}{32 \pi^{2}}, \tau(t)=\sin t
$$

and $p(t)=\cos t$. It is then easy to verify that all the assumptions in Theorem 1 are satisfied with $D=2$, and $K=\frac{1}{33 \pi^{2}}, M=\frac{1}{32 \pi^{2}}, a=\frac{1}{2}$ periodic solution. 
Corollary 2 Suppose there are positive constants $M, D$, and nonnegative constant $K$ such that

$$
\begin{aligned}
& \text { [A] }|f(x)| \leq \frac{K}{2} \text {, for any } x \in R, \\
& \text { [B] } x g(x)>0 \text { and }|g(x)|>K|x| \text { for }|x| \geq D, \\
& \lim _{x \rightarrow-\infty} \frac{g(x)}{x} \leq M \text {, and } \\
& \text { [D] } 8 \pi^{2}(K+2 M)<1 .
\end{aligned}
$$

Then equation (2) has at least one $2 \pi$-periodic solution $x(t)$ and there exists a constant $R^{*}$ such that $|x(t)| \leq R^{*},\left|x^{\prime}(t)\right| \leq R^{*}$.

Corollary 3 Suppose there are positive constants $M, D$, and nonnegative constant $K$ such that

$$
\begin{aligned}
& \text { [A] }|f(x)| \leq \frac{K}{2} \text {, for any } x \in R, \\
& {[\mathrm{~B}] \quad x g(x)>0 \text { and }|g(x)|>K|x| \text { for }|x| \geq D,} \\
& \lim _{x \rightarrow+\infty} \frac{g(x)}{x} \leq M \text {, and } \\
& \text { [D] } 8 \pi^{2}(K+2 M)<1 .
\end{aligned}
$$

Then equation (2) has at least one $2 \pi$-periodic solution $x(t)$ and there exists a constant $R^{*}$ such that $|x(t)| \leq R^{*},\left|x^{\prime}(t)\right| \leq R^{*}$.

\section{REFERENCES}

[1] R. E. Gaines and J. L. Mawhin, Coincidence degree and nonlinear differential equations, Lecture Notes in Math., No. 568, Springer-Verlag, 1977.

[2] K. Deimling, Nonlinear Functional Analysis, Springer-Verlag, 1985.

[3] F. Zanolin, Periodic solutions for differential systems of Rayleigh type, Rend. Istit. Mat. Univ. Trieste, 12(1/2), pp. 69-77,1980.

[4] S. Invernizzi and F. Zanolin, Periodic solutions of a differential delay equation of Rayleigh type, Rend. Sem. Mat. Univ. Padova 61, pp. 115124, 1979.

[5] F. Liu, Existence of periodic solutions to a class of second-order nonlinear differential equations, Acta Math. Sinica 33(2),pp. 260-269, 1990.

[6] P. Omari and G.Villari, Periodic solutions of the Rayleigh equations with damping of definite sign, Atti. Accad. Naz. Lincei Cl. Sci. Fis,Mat. Natur. 1(1), pp. 29-35, 1990.

[7] G. Wang, S. Cheng, A Priori Bounds for Periodic Solutions of a Delay Rayleigh Equation, Applied Mathematics Letters, Vol. 12, pp. 41-44, 1999. 